Sharif University of Technology
Scientia Iranica
Transactions E: Industrial Engineering
http://scientiairanica.sharif.edu

Research Note

\title{
Efficient estimation of Pareto model using modified maximum likelihood estimators
}

\author{
S. Haider Bhatti*, S. Hussain, T. Ahmad, M. Aftab, M.A. Raza, and M. Tahir \\ Department of Statistics, Government College University, Faisalabad, Pakistan.
}

Received 9 June 2017; received in revised form 17 September 2017; accepted 6 June 2018

\author{
KEYWORDS \\ Maximum likelihood \\ estimation; \\ Mean square error; \\ Modified estimators; \\ Pareto distribution; \\ Total relative \\ deviation.
}

\begin{abstract}
In this article, we propose some modifications to the maximum likelihood estimation for estimating the parameters of the Pareto distribution and evaluate the performance of these modified estimators in comparison with the existing maximum likelihood estimators. Total Relative Deviation (TRD), Total Mean Square Error (TMSE), and Stein Loss Function (SLF) were used as performance indicators of goodness of fit analysis. The modified and traditional estimators were compared for different sample sizes and different parameter combinations using a Monte Carlo simulation in R-language. We concluded that the modified maximum likelihood estimator based on expectation of empirical Cumulative Distribution Function (CDF) of first-order statistic performed much better than the traditional ML estimator and other modified estimators based on median and coefficient of variation. The superiority of the mentioned estimator was independent of sample size and choice of true parameter values. The simulation results were further corroborated by employing the proposed estimation strategies for two real-life datasets.

(C) 2019 Sharif University of Technology. All rights reserved.
\end{abstract}

\section{Introduction}

Pareto distribution is one of the most important lifetime distributions. It was developed by Pareto [1] on the basis of the law of income distribution. The two-parameter Pareto distribution is commonly used to model uneven distribution of wealth among individual units in society [2]. It has wide applications in economic studies as it plays a vital role in the investigation into several economic phenomena [3]. However, it is not limited to application in economics and has also been applied in many other disciplines [4,5]. In recent times, it has been used to study the ozone levels in the uppermost atmosphere, tensile strength of nylon

\footnotetext{
*. Corresponding author. E-mail address: sajjad.haider@gcuf.edu.pk (S. Haider Bhatti)
}

doi: $10.24200 /$ sci. 2018.20107 carpet fibers, occurrence of natural resources, insurance risks and the commercial features, etc. Burroughs and Tebbens [6] discussed some applications of the Pareto distribution in modeling the data related to earthquakes, forestry fire areas, and oil and gas in different field sizes. Different variants of Pareto distribution like generalized and transmuted forms have also been discussed in the literature with practical applicability $[7,8]$.

The parameter estimation of Pareto distribution has been carried out with different estimation methods available in the literature. Quandt [9] derived the algebraic expressions for different methods of estimation like method of moments, method of maximum likelihood, quantiles method, and least squares method. Afify [10] derived the recurrence relations and estimated the parameters by moments of order statistics for Pareto distribution. Lu and Tao [11] considered weighted least squares method for estimating the parameters of Pareto distribution. Their results 
showed that both maximum likelihood and weighted least square estimators performed almost identically.

Maximum likelihood estimation is considered as the most important analytical technique for estimating the parameters of any probability distribution. Pobočíková and Sedliačková [12] compared four methods for parameters estimation of Weibull distribution, namely least squares, weighted least squares, maximum likelihood, and method of moments. The numerical results indicated that the method of moments and maximum likelihood provided equivalent results, but they recommended maximum likelihood because of its optimal properties. Similar results have been documented in favor of maximum likelihood estimation for exponential-Pareto distribution [13] and Generalized Pareto distribution [14].

In the literature on estimation of parameters, different modifications have been proposed to the standard estimation techniques. Cohen and Whitten [15] derived the modified moment estimators and modified maximum likelihood estimators for threeparameter Weibull distribution. Most of their modifications were based on first-order statistic. Numerical evaluations have shown that the modified estimators provide higher accuracy than traditional methods. Iwase and Kanefuji [16] studied the modified maximum likelihood estimators and modified moment estimators for the Log-normal distribution with shifted unknown origin. Lalitha and Mishra [17] suggested the modified maximum-likelihood estimation for scale-parameter of the Rayleigh distribution. Modifications to maximum likelihood estimation and moments methods have also been found better than traditional estimators for twoparameter exponential distribution [18]. Similarly, for Power Function distribution, Zaka and Akhter [19] suggested some modifications to the method of maximum likelihood, method of moments, and method of percentile estimation.

Keeping in view the importance of Pareto distribution and maximum likelihood method as well as the superiority of modified maximum likelihood estimation for different distributions in the recent literature, the present study is focused on deriving the modified maximum likelihood estimators for Pareto distribution. The derived modifications have been compared with traditional maximum likelihood estimators using some common performance indicators.

The rest of the article is structured as follows: Section 2 presents different properties of Pareto distribution. Section 3 provides a brief review of methods and derivations performed and the performance indices used for comparison. Section 4 describes the simulation procedure employed. Sections 5 and 6 present the results and discussion on simulation study and real-life applications, respectively. Finally, Section 7 concludes the article.

\section{Properties of Pareto distribution}

The Pareto distribution can be expressed with shape $(\alpha)$ and scale $(\beta)$ parameters. The values of these parameters must be positive. Let $t_{1}, t_{2}, t_{3}, \ldots, t_{n}$ be a random sample from two-parameter Pareto distribution; then, probability density function (pdf) is given as:

$$
f(t ; \alpha, \beta)=\frac{\alpha \beta^{\alpha}}{t^{\alpha+1}} \quad t \geq \beta \quad \text { and } \quad \alpha, \beta>0 .
$$

Different properties of Pareto distribution are given below:

The Cumulative Distribution Function (CDF) of Pareto distribution:

$$
F(t)=P(T \leq t)=1-\left(\frac{\beta}{t}\right)^{\alpha} .
$$

Survival function:

$$
S(t)=1-F(t)=P(T>t)=\left(\frac{\beta}{t}\right)^{\alpha} .
$$

Hazard function:

$$
h(t)=\frac{f(t)}{S(t)}=\left(\frac{\alpha}{t}\right) .
$$

Entropy of Pareto distribution:

$$
\text { Entropy }=\log \left[\left(\frac{\beta}{\alpha}\right) e^{\left(1+\frac{1}{\alpha}\right)}\right] \text {. }
$$

Mean and variance of Pareto distribution:

$$
\begin{aligned}
& \text { Mean }=\frac{\alpha \beta}{\alpha-1}, \quad \alpha>1, \\
& \text { Variance }=\frac{\alpha \beta^{2}}{(\alpha-2)(\alpha-1)^{2}}, \quad \alpha>2 .
\end{aligned}
$$

Coefficient of variation:

$$
C V=\frac{1}{\sqrt{\alpha(\alpha-2)}} .
$$

Median:

$$
\int_{-\infty}^{m} f(t) d t=\frac{1}{2} \quad \Rightarrow \quad \text { Median }=\beta(2)^{1 / \alpha} .
$$

Harmonic mean:

$$
H M=\beta\left(1+\frac{1}{\alpha}\right) \text {. }
$$

The geometric mean:

$$
G M=\beta e^{1 / \alpha} .
$$

Mean deviation: 


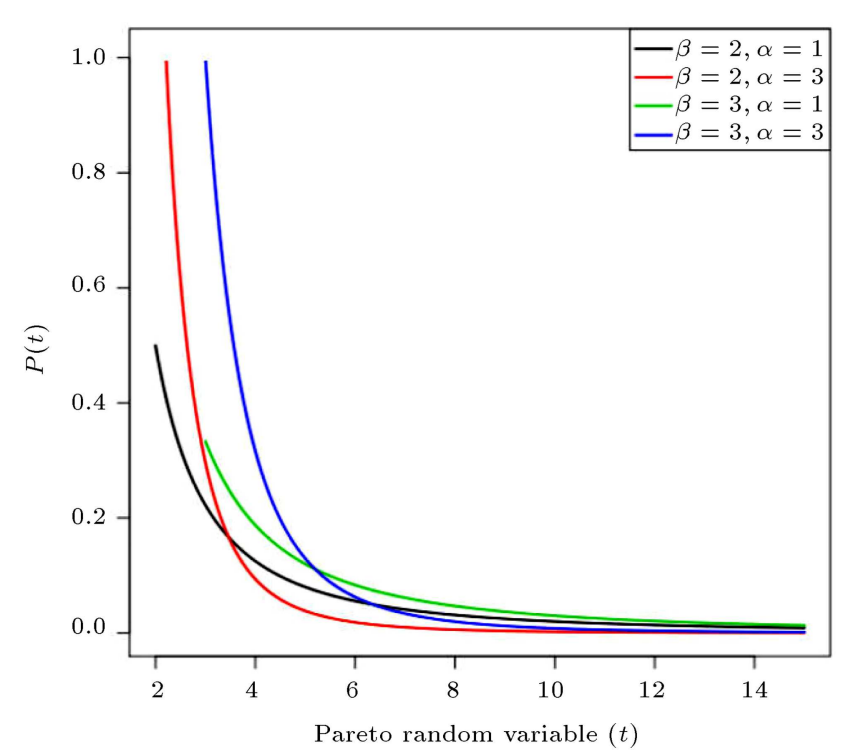

Figure 1. Pareto distribution with different parameter combinations.

$$
M D=2 \beta(\alpha-1)\left(1-\frac{1}{\alpha}\right)^{\alpha-1} .
$$

The $r$ th moments about origin:

$$
\mu_{r}{ }^{\prime}=\frac{\alpha \beta^{r}}{\alpha-r}, \quad \text { where } \quad r<\alpha
$$

Coefficient of skewness:

$$
\gamma_{1}=\frac{2(\alpha+1)}{(\alpha-3)} \sqrt{\frac{(\alpha-2)}{\alpha}}, \quad \alpha>3 .
$$

Measure of kurtosis:

$$
\gamma_{2}=\frac{3(\alpha-2)\left(3 \alpha^{2}+\alpha+2\right)}{\alpha(\alpha-3)(\alpha-4)}, \quad \alpha>4 .
$$

Moment generating function:

$$
M(r ; \alpha, \beta)=E\left[e^{r t}\right]=\alpha(-r \beta)^{\alpha} \Gamma(-\alpha,-r \beta) .
$$

Characteristics function:

$$
\varphi(r ; \alpha, \beta)=\alpha(-i \beta r)^{\alpha} \Gamma(-\alpha,-i r \beta) .
$$

Shape of Pareto distribution with different combinations of scale and shape parameters is depicted in Figure 1.

\section{Methodology}

In the current study, we have derived some modifications through the maximum likelihood estimation approach and compared them with the traditional one. The proposed modifications are based on median, coefficient of variation, and expectation of empirical CDF of first-order statistic of Pareto distribution.

\subsection{Maximum Likelihood (ML) estimation}

The method of ML estimation was introduced by Fisher [20]. This method is widely used for parameter estimation. The ML estimators are generally unbiased and possess optimal properties.

\subsection{Maximum likelihood estimation of Pareto distribution}

Let $t_{1}, t_{2}, \ldots, t_{n}$ be a random sample from Pareto distribution. The Probability density function of the Pareto distribution is:

$$
f(t ; \alpha, \beta)= \begin{cases}\frac{\alpha \beta^{\alpha}}{t^{\alpha+1}} ; & t \geq \beta, \quad \beta>0, \quad \alpha>0 \\ 0 & \text { Elsewhere, }\end{cases}
$$

where $\beta$ is shape and $\alpha$ is the scale parameter commonly denoted by $t_{i} \sim \operatorname{Pareto}(\beta, \alpha)$. The $\log$ likelihood function is:

$$
\begin{aligned}
\ln L= & \left(n \ln \alpha+n \alpha \ln \beta-(\alpha+1) \sum_{i=1}^{n} \ln t_{i}\right) \\
& \mathrm{I}\left\{\min _{i} t_{i}>\beta\right\} .
\end{aligned}
$$

Differentiating Eq. (1) with respect to " $\alpha$ " leads to:

$$
\frac{n}{\alpha}+n \ln \beta-\sum_{i=1}^{n} \ln t_{i}=0
$$

hence, ML estimators of $\alpha$ and $\beta$ (by direct maximization) are:

$$
\begin{aligned}
& \hat{\alpha}=\frac{n}{\sum_{i=1}^{n} \ln t_{i}-n \ln \beta}, \\
& \hat{\beta}=t_{(1)},
\end{aligned}
$$

where $t_{(1)}$ is the lowest value in the sample.

\subsection{Modified maximum likelihood estimator-I} For the first modification to the ML method, we followed Cohen and Whitten [15], Rashid and Akhter [18], and Zaka and Akhter [19] who derived the modified ML estimators for Weibull, exponential, and power function distributions, respectively. In this modification, we use median of Pareto distribution and Eq. (2).

The median of Pareto distribution is:

$$
\begin{aligned}
& \tilde{t}=\beta 2^{\frac{1}{\alpha}}, \\
& \beta=\frac{\tilde{t}}{2^{\frac{1}{\alpha}}} .
\end{aligned}
$$

Putting the value of $\beta$ in Eq. (2), we get the first modified ML estimators of Pareto distribution as: 


$$
\begin{aligned}
& \hat{\alpha}=\frac{n(1-\ln 2)}{\sum_{i=1}^{n} \ln t_{i}-n \ln \tilde{t}} \\
& \hat{\beta}=\frac{\tilde{t}}{2^{1 / \hat{\alpha}}} .
\end{aligned}
$$

In the following, we name them as ML-I.

\subsection{Modified maximum likelihood estimator-II}

For the second modification to the method of ML estimation, we followed Cohen and Whitten [21], Rashid and Akhter [18], and Zaka and Akhter [19]. They derived the modified ML estimators for gamma, exponential, and power function distributions, respectively. This modification employs the coefficient of variation and Eq. (2).

The coefficient of variation of Pareto distribution is given as:

$$
C . V .=\frac{1}{\sqrt{\alpha(\alpha-2)}}, \quad \alpha>2,
$$

from Eq. (2):

$$
\beta=\exp \left(\frac{\sum_{i=1}^{n} \ln t_{i}-\frac{n}{\alpha}}{n}\right),
$$

and from Eq. (9):

$$
\begin{aligned}
& \frac{s}{\bar{t}}=\frac{1}{\sqrt{\alpha(\alpha-2)}} \\
& \Rightarrow \quad \alpha(\alpha-2)=\frac{\bar{t}^{2}}{s^{2}} \\
& \hat{\alpha}=1+\sqrt{1+\frac{\bar{t}^{2}}{s^{2}}} .
\end{aligned}
$$

Putting $\hat{\alpha}$ from Eq. (11) in Eq. (10), we get the estimator of $\beta$ as:

$$
\hat{\beta}=\exp \left(\frac{\sum_{i=1}^{n} \ln t_{i}-\frac{n s}{s+\sqrt{s^{2}+\bar{t}^{2}}}}{n}\right) .
$$

Thus, Eqs. (11) and (12) are the second modified ML estimators of $\alpha$ and $\beta$. In the following, we name them as ML-II

\subsection{Modified maximum likelihood estimator-III}

For the third modification to the ML method, we followed Rashid and Akhter [18]. They derived the modified ML estimator for exponential distribution. This modification is based on Eq. (2) and expectation of empirical CDF of first-order statistic of Pareto distribution. Following Cohen and Whitten [15], Rashid and Akhter [18], Zaka and Akhter [19], and Cohen and Whitten [21], expectation of empirical CDF of firstorder statistic is defined as:

$$
E\left[F\left(t_{(1)}\right)\right]=\frac{1}{n+1} \text {. }
$$

Hence, expectation of empirical CDF of first-order statistic of the Pareto distribution is:

$$
\begin{aligned}
& \frac{1}{n+1}=1-\left(\frac{\beta}{t_{(1)}}\right)^{\alpha}, \\
& \beta=t_{(1)}\left(\frac{n}{n+1}\right)^{\left(\frac{1}{\alpha}\right)} .
\end{aligned}
$$

Putting $\beta$ from Eq. (15) in Eq. (2) and solving it for $\alpha$, we get:

$$
\hat{\alpha}=\frac{n[1+\ln (n)-\ln (n+1)]}{\sum_{i=1}^{n} \ln t_{i}-n \ln \left(t_{(1)}\right)}
$$

Eq. (15) becomes:

$$
\hat{\beta}=t_{(1)}\left(\frac{n}{n+1}\right)^{\left(\frac{1}{\hat{\alpha}}\right)} \text {. }
$$

Thus, Eqs. (15) and (16) provide the third modified ML estimators of Pareto distribution. In the following, we call them ML-III.

\subsection{Performance indices}

For comparing the performances of traditional ML estimators and the proposed modified ML estimators, three performance indices, namely Total Mean Square Error (TMSE), Total Relative Deviation (TRD), and Stein Loss Function (SLF), are used. These indices provide precision and accuracy of estimators. These measures are frequently used in the literature as performance criteria for the comparison of estimators [18,19,22-25].

TMSE for the parameter vector is calculated as:

$$
\mathrm{TMSE}=
$$

$$
\frac{\sum_{r=1}^{\mathrm{R}}\left(\left[\begin{array}{c}
\hat{\beta}_{r} \\
\hat{\alpha}_{r}
\end{array}\right]-\left[\begin{array}{l}
\beta_{r} \\
\alpha_{r}
\end{array}\right]\right)^{\prime}\left(\left[\begin{array}{l}
\hat{\beta}_{r} \\
\hat{\alpha}_{r}
\end{array}\right]-\left[\begin{array}{l}
\beta_{r} \\
\alpha_{r}
\end{array}\right]\right)}{R}
$$

where $R$ is the number of replications, which reduces to:

$$
\begin{aligned}
\mathrm{TMSE} & =\frac{\sum_{r=1}^{\mathrm{R}}\left[\left(\hat{\beta}_{r}-\beta\right)^{2}+\left(\hat{\alpha}_{r}-\alpha\right)^{2}\right]}{R} \\
& =\operatorname{MSE}(\hat{\beta})+\operatorname{MSE}(\hat{\alpha}) .
\end{aligned}
$$

The following expression is used for the calculation of TRD: 


$$
T R D=\left|\frac{E(\hat{\alpha})-\alpha}{\alpha}\right|+\left|\frac{E(\hat{\beta})-\beta}{\beta}\right|,
$$

where $\alpha$ and $\beta$ are the true parameters.

Stein Loss Function (SLF) is defined by James and Stein [26] as:

$$
S L F=\frac{\hat{\theta}}{\theta}-\log \left(\frac{\hat{\theta}}{\theta}\right) .
$$

\section{Numerical evaluation}

A simulation study is conducted to assess the performances of modified ML estimators proposed in the current article. This comparison is done for different sample sizes $(n=20,50,100,200)$ and different parameter combinations $(\beta=1 \alpha=3 ; \beta=1 \alpha=4$; $\beta=2 \alpha=3 ; \beta=2 \alpha=4)$. The random samples are drawn such that if $\mathrm{U}_{i} \sim \operatorname{Uniform}(0,1)$, then $t_{i}=\beta\left(1-U_{i}\right)^{-1 / \alpha}$ is a Pareto random variable with parameters $(\beta, \alpha)$. All the simulation results are based on 10,000 replications using R-language [27].

\section{Results and discussion}

The comparison of different estimators based on TMSE and TRD is given in Tables 1-4 for different samples sizes and parameter combinations. From Table 1, for $n=20$, it can be observed that ML-III (TMSE =
$0.597061 ; \mathrm{TRD}=0.054101 ; \mathrm{SLF}=0.028364)$ provides more precise and efficient estimates than ML (TMSE $=0.735645 ; \mathrm{TRD}=0.124546 ; \mathrm{SLF}=0.032513)$, ML-I (TMSE $=6628.903 ;$ TRD $=0.225408 ;$ SLF $=$ 0.033465 ), and ML-II (TMSE $=2.299222 ;$ TRD $=$ 0.420594; SLF $=0.088004)$, respectively. For $n=50$, ML-III (TMSE $=0.207959 ;$ TRD $=0.02242 ;$ SLF $=0.010825)$ gives estimates which are more efficient and close to true parameters than the estimates of $\mathrm{ML}(\mathrm{TMSE}=0.228458 ; \mathrm{TRD}=0.04969 ; \mathrm{SLF}=$ 0.0115), ML-I (TMSE = 226.6148; TRD = 0.215618; $\mathrm{SLF}=0.01824)$, and ML-II $(\mathrm{TMSE}=0.881554 ; \mathrm{TRD}$ $=0.257158 ; \mathrm{SLF}=0.039857)$, respectively. Similarly, for the sample size of 100, ML-III performs better than traditional ML and two modified ML estimators in terms of TMSE $(0.097426,1.252958,0.466227$, and 0.092797 for ML, ML-I, ML-II, and ML-III, respectively) as well as in terms of TRD (0.023491, 0.106291, 0.179633 , and 0.010054 for ML, ML-I, ML-II, and ML-III, respectively) and SLF (0.005158, 0.049679, 0.022946 , and 0.005002 for ML, ML-I, ML-II, and ML-III, respectively). Finally, for $n=200$, ML-III also performs better than other competing estimators considered. ML-III gives TMSE $=0.04677$, while ML, ML-I, and ML-II give TMSE $=0.04795,0.407725$, and 0.263359 , respectively. Similarly, TRD is computed at 0.005321 for ML-III compared to TRD $=0.012011$, 0.046269 , and 0.127321 for ML, ML-I, and ML-II, respectively. For $n=200$, in terms of SLF, the

\begin{tabular}{|c|c|c|c|c|c|c|}
\hline$n$ & Method & $\boldsymbol{E}(\hat{\boldsymbol{\beta}})$ & $\boldsymbol{E}(\hat{\boldsymbol{\alpha}})$ & TMSE & TRD & SLF \\
\hline \multirow{4}{*}{20} & ML & 1.017117 & 3.322288 & 0.735645 & 0.124546 & 0.032513 \\
\hline & ML-I & 1.044508 & 3.5427 & 6628.903 & 0.225408 & 0.033465 \\
\hline & ML-II & 1.07382 & 4.040321 & 2.299222 & 0.420594 & 0.088004 \\
\hline & ML-III & 1.000703 & 3.160193 & 0.597061 & 0.054101 & 0.028364 \\
\hline \multirow{4}{*}{50} & ML & 1.006673 & 3.12905 & 0.228458 & 0.04969 & 0.0115 \\
\hline & ML-I & 1.017977 & 3.592925 & 226.6148 & 0.215618 & 0.01824 \\
\hline & ML-II & 1.049072 & 3.624257 & 0.881554 & 0.257158 & 0.039857 \\
\hline & ML-III & 1.000058 & 3.067086 & 0.207959 & 0.02242 & 0.010825 \\
\hline \multirow{4}{*}{100} & ML & 1.003304 & 3.060562 & 0.097426 & 0.023491 & 0.005158 \\
\hline & ML-I & 1.008047 & 3.294731 & 1.252958 & 0.106291 & 0.049679 \\
\hline & ML-II & 1.036149 & 3.430452 & 0.466227 & 0.179633 & 0.022946 \\
\hline & ML-III & 0.999982 & 3.030108 & 0.092797 & 0.010054 & 0.005002 \\
\hline \multirow{4}{*}{200} & ML & 1.001657 & 3.031062 & 0.04795 & 0.012011 & 0.0026 \\
\hline & ML-I & 1.003702 & 3.127701 & 0.407725 & 0.046269 & 0.020397 \\
\hline & ML-II & 1.0265 & 3.302463 & 0.263359 & 0.127321 & 0.013839 \\
\hline & ML-III & 0.999994 & 3.015944 & 0.04677 & 0.005321 & 0.00256 \\
\hline
\end{tabular}

Table 1. Comparison of ML, ML-I, ML-II, and ML-III for $\beta=1$ and $\alpha=3$. 
Table 2. Comparison of ML, ML-I, ML-II, and ML-III for $\beta=1$ and $\alpha=4$.

\begin{tabular}{|c|c|c|c|c|c|c|}
\hline$n$ & Method & $\boldsymbol{E}(\hat{\boldsymbol{\beta}})$ & $E(\hat{\boldsymbol{\alpha}})$ & TMSE & TRD & SLF \\
\hline \multirow{4}{*}{20} & ML & 1.012651 & 4.447734 & 1.380098 & 0.124585 & 0.033644 \\
\hline & ML-I & 1.028744 & 6.971119 & 4878.77 & 0.771524 & 0.185629 \\
\hline & ML-II & 1.039263 & 5.095194 & 3.419774 & 0.313061 & 0.073896 \\
\hline & ML-III & 1.000401 & 4.230728 & 1.120442 & 0.058083 & 0.029338 \\
\hline \multirow{4}{*}{50} & ML & 1.00496 & 4.164452 & 0.388137 & 0.046073 & 0.011032 \\
\hline & ML-I & 1.011489 & 4.87495 & 132.8122 & 0.230226 & 0.127452 \\
\hline & ML-II & 1.02419 & 4.598745 & 1.236833 & 0.173876 & 0.032191 \\
\hline & ML-III & 0.999997 & 4.081985 & 0.353632 & 0.020499 & 0.010405 \\
\hline \multirow{4}{*}{100} & ML & 1.002504 & 4.078189 & 0.177935 & 0.022051 & 0.005277 \\
\hline & ML-I & 1.006098 & 4.405924 & 5.563232 & 0.107579 & 0.050877 \\
\hline & ML-II & 1.016198 & 4.374583 & 0.625432 & 0.109844 & 0.017776 \\
\hline & ML-III & 1.000011 & 4.037609 & 0.169828 & 0.009413 & 0.005129 \\
\hline \multirow{4}{*}{200} & ML & 1.001261 & 4.040215 & 0.084378 & 0.011315 & 0.002571 \\
\hline & ML-I & 1.002893 & 4.173587 & 0.700546 & 0.046289 & 0.019442 \\
\hline & ML-II & 1.010139 & 4.233041 & 0.344255 & 0.068399 & 0.010534 \\
\hline & ML-III & 1.000013 & 4.020065 & 0.082338 & 0.005029 & 0.002532 \\
\hline
\end{tabular}

Table 3. Comparison of ML, ML-I, ML-II, and ML-III for $\beta=2$ and $\alpha=3$.

\begin{tabular}{|c|c|c|c|c|c|c|}
\hline$n$ & Method & $\boldsymbol{E}(\hat{\boldsymbol{\beta}})$ & $E(\hat{\alpha})$ & TMSE & TRD & SLF \\
\hline \multirow{4}{*}{20} & ML & 2.032983 & 3.334168 & 0.773015 & 0.127881 & 0.033618 \\
\hline & ML-I & 2.083013 & 5.50672 & 6222.556 & 0.87708 & 0.179457 \\
\hline & ML-II & 2.14457 & 4.033481 & 2.292159 & 0.416779 & 0.086644 \\
\hline & ML-III & 2.000253 & 3.171493 & 0.626941 & 0.057291 & 0.029286 \\
\hline \multirow{4}{*}{50} & ML & 2.01352 & 3.1227 & 0.22508 & 0.04766 & 0.011338 \\
\hline & ML-I & 2.034664 & 3.998999 & 459.6621 & 0.350332 & 0.044614 \\
\hline & ML-II & 2.09871 & 3.619203 & 0.890822 & 0.255756 & 0.039591 \\
\hline & ML-III & 2.000264 & 3.060863 & 0.205327 & 0.02042 & 0.010705 \\
\hline \multirow{4}{*}{100} & ML & 2.006758 & 3.062779 & 0.101324 & 0.024305 & 0.005341 \\
\hline & ML-I & 2.021307 & 3.354927 & 7.999015 & 0.128963 & 0.061298 \\
\hline & ML-II & 2.073637 & 3.440908 & 0.495619 & 0.183788 & 0.023805 \\
\hline & ML-III & 2.000117 & 3.032304 & 0.096454 & 0.010826 & 0.005177 \\
\hline \multirow{4}{*}{200} & ML & 2.003418 & 3.030724 & 0.047555 & 0.01195 & 0.00257 \\
\hline & ML-I & 2.008491 & 3.132881 & 0.416321 & 0.048539 & 0.020534 \\
\hline & ML-II & 2.053206 & 3.304872 & 0.278185 & 0.128227 & 0.014233 \\
\hline & ML-III & 2.000091 & 3.015609 & 0.046379 & 0.005248 & 0.00253 \\
\hline
\end{tabular}


Table 4. Comparison of ML, ML-I, ML-II, and ML-III for $\beta=2$ and $\alpha=4$.

\begin{tabular}{|c|c|c|c|c|c|c|}
\hline$n$ & Method & $E(\hat{\beta})$ & $E(\hat{\alpha})$ & TMSE & TRD & SLF \\
\hline \multirow{4}{*}{20} & ML & 2.025154 & 4.443085 & 1.352143 & 0.123348 & 0.033198 \\
\hline & ML-I & 2.058967 & 3.974279 & 31993.43 & 0.035914 & 0.074343 \\
\hline & ML-II & 2.080093 & 5.108112 & 3.467063 & 0.317075 & 0.074179 \\
\hline & ML-III & 2.000645 & 4.226306 & 1.096509 & 0.056899 & 0.02895 \\
\hline \multirow{4}{*}{50} & ML & 2.009922 & 4.167355 & 0.396775 & 0.0468 & 0.011231 \\
\hline & ML-I & 2.026314 & 5.088347 & 131.543 & 0.285244 & 0.046526 \\
\hline & ML-II & 2.04903 & 4.608011 & 1.271466 & 0.176518 & 0.032646 \\
\hline & ML-III & 2.000001 & 4.084831 & 0.361413 & 0.021208 & 0.010589 \\
\hline \multirow{4}{*}{100} & ML & 2.00497 & 4.08406 & 0.177909 & 0.0235 & 0.005297 \\
\hline & ML-I & 2.013689 & 4.418893 & 2.371643 & 0.111568 & 0.048882 \\
\hline & ML-II & 2.03278 & 4.387988 & 0.650319 & 0.113387 & 0.018412 \\
\hline & ML-III & 1.999992 & 4.043422 & 0.169322 & 0.01086 & 0.005135 \\
\hline \multirow{4}{*}{200} & $\mathrm{ML}$ & 2.002537 & 4.039 & 0.084804 & 0.011018 & 0.002585 \\
\hline & ML-I & 2.006899 & 4.184829 & 0.729367 & 0.049657 & 0.01999 \\
\hline & ML-II & 2.020911 & 4.238906 & 0.358476 & 0.070182 & 0.010864 \\
\hline & ML-III & 2.00004 & 4.018856 & 0.082804 & 0.004734 & 0.002548 \\
\hline
\end{tabular}

results show superiority of ML-III as it has lower SLF value than other competing estimators $(\mathrm{SLF}=0.00256$ for ML-III compared to SLF $=0.0026,0.020397$, and 0.013839 for ML, ML-I, and ML-II, respectively).

From Tables 2, 3, and 4, it is evident that the parameter estimates of ML-III are more precise and efficient than those of the traditional ML and other modified estimators (ML-I and ML-II) for all sample sizes and all parameter combinations. The TMSE and TRD, in case of ML-III, are smaller than the results of other estimators. ML-I performs the worst for small samples; however, when the sample size becomes large, ML-I estimates get closer to the actual parameters. During computations, similar results are observed for sample sizes of up to 1000 for all parameter combinations. These results have been skipped to avoid redundancy.

It is also worth mentioning that ML-III performs better for all sample sizes and for all parameter combinations. However, its superiority over traditional ML estimators has a decreasing tendency with growing sample size.

\section{Real data applications}

In addition to the simulation study, the proposed modified estimators are compared using two real-life datasets. The first example is taken from Clark [28], which was also used by Kantar [29], and consists of
21 observations of the data on the number of deaths in major earthquakes during 1900-2011 as published by the U.S. Geological Survey. The second example is taken from Beirliant et al. [30] consisting of 142 values of fire damage claims (in 1000's of Norwegian Krones) in Norway during 1975. The same dataset has also been used by Munir et al. [2] and Obradović [31] for comparing different estimators as well as the efficiency of goodness of fit tests in case of Pareto distribution.

The TMSE and TRD cannot be used as performance measures in real-life data, because, unlike in the simulation, the true parameters are not known. Thus, for the comparison of the performance of estimators in real-life situations, we use two other measures. The first one is values of the test statistic of KolmogorovSmirnov (KS) goodness of fit test $[32,33]$ assuming a Pareto distribution with given parameters estimated from any of the four methods. The second one is the Sum of Squared Differences (SSD) between observed (sample) distribution function, $S\left(t_{i}\right)$, and expected distribution function, $\hat{F}\left(t_{i}\right)$, with parameters estimated from any method. This squared difference is defined as:

$$
S S D=\sum_{i=1}^{n}\left\{S\left(t_{i}\right)-\hat{F}\left(t_{i}\right)\right\}^{2} .
$$

Both of the above measures are based on choosing the combination of parameter estimates that provide a better fit to the observed data. The results from reallife applications are presented in Table 5 . 
Table 5. Comparison of estimators for real-life examples.

\begin{tabular}{cccccc}
\hline & Method & $\hat{\boldsymbol{\beta}}$ & $\hat{\boldsymbol{\alpha}}$ & $\boldsymbol{K}-\boldsymbol{S}$ test (statistic) & SSD \\
\hline \multirow{2}{*}{$\begin{array}{c}\text { Mxample-1 } \\
n=21\end{array}$} & ML-I & 20085 & 0.903376 & 0.152473 & 0.046585 \\
& ML-II & 40353.96 & 2.443539 & 0.404264 & 0.715023 \\
& ML-III & 19029.02 & 0.861351 & 0.150542 & 0.040972 \\
& & & & & \\
Example-2 & ML & 500 & 1.19403 & 0.054996 & 0.053872 \\
$n=142$ & ML-I & 532.6737 & 1.291658 & 0.097218 & 0.268736 \\
& ML-II & 702.0035 & 2.007348 & 0.352113 & 3.420614 \\
& ML-III & 497.0494 & 1.185651 & 0.051481 & 0.048543 \\
\hline
\end{tabular}

From the application of the considered estimation strategies to the first real-life example, it is evident that ML-III provides a more precise fit to the actual data in terms of KS-test statistic (0.150542 for ML-III compared to $0.152473,0.404264$, and 0.443881 for ML, ML-I, and ML-II, respectively) as well as in terms of the sum of squared differences between observed and expected CDFs (SSD $=0.040972$ for ML-III compared to $\mathrm{SSD}=0.046585,0.715023$, and 1.020271 for ML, ML-I, and ML-II, respectively). Similar results are obtained for the second real-life example. Hence, both real data applications corroborate our simulation results presented in the previous section.

\section{Conclusion}

The study dealt with the parameter estimation of Pareto distribution with some modified ML estimators. We derived the algebraic expressions for three modified ML estimators. The proposed modifications were based on median, coefficient of variation, and expectation of empirical CDF of first-order statistic. A Monte Carlo simulation study based on 10,000 replications was performed with different sample sizes and different parameter combinations. From the results, it could be concluded that modified estimator based on expectation of empirical CDF of first-order statistic (ML-III) was more precise and efficient than the traditional and other modified ML estimators for all the sample sizes and parameter combinations considered. The results were further confirmed by applying the proposed estimation strategies to two real-life examples.

\section{References}

1. Pareto, V. "The new theories of economics", J. Polit. Econ., 5(4), pp. 485-502 (1897).

2. Munir, R., Saleem, M., Aslam, M., and Ali, S. "Comparison of different methods of parameters estimation for Pareto model", Casp. J. Appl. Sci. Res., 2(1), pp. 45-56 (2013).
3. Arnold, B.C. Encyclopaedia of Statistical Sciences, John Wiley (2008).

4. Abdel-All, N.H., Mahmoud, M.A.W., and Abd-Ellah, H.N. "Geometrical properties of Pareto distribution", Appl. Math. Comput., 145(2), pp. 321-339 (2003).

5. Sankaran, P.G. and Nair, M.T. "On finite mixture of Pareto distributions", Calcutta Stat. Assoc. Bull., 57(1-2), pp. 225-226 (2005).

6. Burroughs, S.M. and Tebbens, S.F. "Upper-truncated power law distributions", Fractals, 9(1), pp. 209-222 (2001).

7. Castillo, E. and Hadi, A.S. "Fitting the generalized Pareto distribution to data", J. Am. Stat. Assoc., 92(440), pp. 1609-1620 (1997).

8. Bourguignon, M., Ghosh, I., and Cordeiro, G.M. "General results for the transmuted family of distributions and new models", J. Probab. Stat., 2016, pp. 1-12 (2016).

9. Quandt, R.E. "Old and new methods of estimation and the Pareto distribution", Metrika, 10(1), pp. 5582 (1966).

10. Afify, E.E. "Order statistics from Pareto distribution", J. Appl. Sci., 6(10), pp. 2151-2157 (2006).

11. Lu, H.-L. and Tao, S.H. "The estimation of Pareto distribution by a weighted least square method", Qual. Quant., 41(6), pp. 913-926 (2007).

12. Pobočíková, I. and Sedliačková, Z. "Comparison of four methods for estimating the Weibull distribution parameters", Appl. Math. Sci., 8(83), pp. 4137-4149 (2014).

13. Shawky, A.I. and Abu-Zinadah, H.H. "Exponentiated Pareto distribution: different method of estimations", Int. J. Contemp. Mathematical Sci., 4(14), pp. 677-693 (2009).

14. Grimshaw, S.D. "Computing maximum likelihood estimates for the generalized Pareto distribution", Technometrics, 35(2), pp. 185-191 (1993).

15. Cohen, A.C. and Whitten, B. "Modified maximum likelihood and modified moment estimators for the three-parameter Weibull distribution", Commun. Stat. Methods, 11(23), pp. 2631-2656 (1982). 
16. Iwase, K. and Kanefuji, K. "Estimation for 3parameter lognormal distribution with unknown shifted origin", Stat. Pap., 35(1), pp. 81-90 (1994).

17. Lalitha, S. and Mishra, A. "Modified maximum likelihood estimation for Rayleigh distribution", Commun. Stat. - Theory Methods, 25(2), pp. 389-401 (1996).

18. Rashid, M.Z. and Akhter, A.S. "Estimation accuracy of exponential distribution parameters", Pakistan J. Stat. Oper. Res., 7(2), pp. 217-232 (2011).

19. Zaka, A. and Akhter, A.S. "Modified moment, maximum likelihood and percentile estimators for the parameters of the power function distribution", Pakistan J. Stat. Oper. Res., 10(4), pp. 361-368 (2014).

20. Fisher, R.A. "On the mathematical foundations of theoretical statistics", Philos. Trans. R. Soc. London. Ser. A, Contain. Pap. a Math. or Phys. Character, 222, pp. 309-368 (1922).

21. Cohen, C.A. and Whitten, B.J. "Modified moment and maximum likelihood estimators for parameters of the three-parameter Gamma distribution", Commun. Stat. Comput., 11(2), pp. 197-216 (1982).

22. Al-Fawzan, M.A., Methods for Estimating the Parameters of the Weibull Distribution, King Abdulaziz City Sci. Technol. (2000).

23. Khalaf, G., Mansson, K., and Shukur, G. "Modified ridge regression estimators", Commun. Stat. - Theory Methods, 42(8), pp. 1476-1487 (2013).

24. Aydin, D. and Şenoğlu, B. "Monte Carlo comparison of the parameter estimation methods for the twoparameter Gumbel distribution", J. Mod. Appl. Stat. Methods, 14(2), pp. 123-140 (2015).

25. Shakeel, M., Haq, M.A. ul, Hussain, I., Abdulhamid, A.M., and Faisal, M. "Comparison of two new robust parameter estimation methods for the power function distribution", PLoS ONE, 11(8), p. e016069 (2016).

26. James, W. and Stein, C. "Estimation with quadratic loss", Proc. Fourth Berkeley Symp. Math. Stat. Probab., 1(1961), pp. 361-379 (1961).

27. Team, R.C.R, A Language and Environment for Statistical Computing, R Foundation for Statistical Computing, Vienna, Austria (2016).

28. Clark, D.R., A Note on the Upper-truncated Pareto Distribution, Casualty Actuarial Society E-Forum, Winter, pp. 1-22 (2013).

29. Kantar, Y.M. "Generalized least squares and weighted least squares estimation methods for distributional parameters", REVSTAT - Stat. J., 13(3), pp. 263-282 (2015).

30. Beirliant, J., Teugels, J.L., and Vynckier, P., Practical Analysis of Extreme Values, Leuven University Press, Leuven, Belgium (1996).

31. Obradović, M. "On Asymptotic efficiency of goodness of fit tests for Pareto distribution based on characterizations", Filomat, 29(10), pp. 2311-2324 (2015).
32. Kolmogorov, A. "On the determination of empirical fit of a distribution" [Sulla determinazione empirica di una legge di distribuzione], G. dell'Istituto Ital. degli Attuari, 4, pp. 83-91 (1933).

33. Smirnov, N.V. "Estimate of deviation between empirical distribution functions in two independent samples", Bull. Moscow Univ., 2, pp. 3-16 (1939).

\section{Biographies}

Sajjad Haider Bhatti received his PhD in Applied Statistics and Econometrics from University of Dijon, France. He received his MSc from the Department of Statistics, Bahauddin Zakariya University, Multan, Pakistan. He is currently working as Assistant Professor in the Department of Statistics, Government College University, Faisalabad, Pakistan. His Research interests include regression diagnostics, modified estimators, and multivariate statistical analysis.

Shahzad Hussain received his MPhil in Statistics from the Department of Statistics, Government College University, Faisalabad, Pakistan. He received his MSc from the Department of Statistics, University of Punjab, Lahore, Pakistan. His research interests are estimation, probability distributions, and extreme value modelling.

Tanvir Ahmad received his $\mathrm{PhD}$ in Statistics from Islamia University Bahawalpur, Pakistan, He received his MSc from the Department of Statistics, Bahauddin Zakariya University, Multan, Pakistan. He was with University of Southampton, UK, as a post-doctoral researcher. He is currently working as Assistant Professor and the Chairman of the Department of Statistics, Government College University, Faisalabad, Pakistan. His research interests include experimental design, response surface methodology, and robust estimation methods.

Muhammad Aftab received his MPhil in Statistics from the Department of Mathematics and Statistics, University of Agriculture, Faisalabad, Pakistan. Also, he received his MSc from the Department of Statistics, Government College University, Faisalabad, Pakistan. He is currently working as a lecturer in the Department of Statistics, Government College University, Faisalabad, Pakistan. His Research interests are categorical data analysis and probability distributions.

Muhammad Ali Raza received his MPhil from the Department of Statistics, University of the Punjab, Lahore, Pakistan. He is currently working as Assistant Professor in the Department of Statistics, Government College University, Faisalabad, Pakistan. His current research interests include applied statistics, parameter 
estimation, and application of probability distribution in statistical quality control.

Muhammad Tahir received his $\mathrm{PhD}$ in Statistics from Quaid-i-Azam University, Islamabad, Pakistan.
He is currently working as Assistant Professor in the Department of Statistics, Government College University, Faisalabad, Pakistan. His research interests are Bayesian statistics, mixture distributions, and mathematical statistics. 